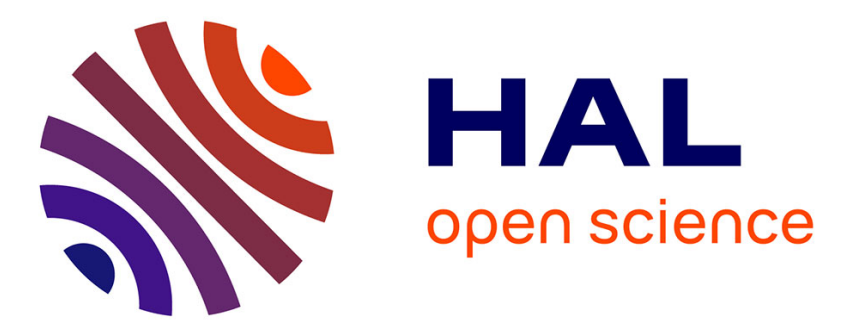

\title{
Muscle fascicle shortening behaviour of vastus lateralis during a maximal force-velocity test
}

\author{
Hugo Hauraix, Sylvain Dorel, Giuseppe Rabita, Gaël Guilhem, Antoine
}

Nordez

\section{- To cite this version:}

Hugo Hauraix, Sylvain Dorel, Giuseppe Rabita, Gaël Guilhem, Antoine Nordez. Muscle fascicle shortening behaviour of vastus lateralis during a maximal force-velocity test. European Journal of Applied Physiology, 2017, 10.1007/s00421-016-3518-4 . hal-01662520

HAL Id: hal-01662520

https: / hal-insep.archives-ouvertes.fr/hal-01662520

Submitted on 13 Dec 2017

HAL is a multi-disciplinary open access archive for the deposit and dissemination of scientific research documents, whether they are published or not. The documents may come from teaching and research institutions in France or abroad, or from public or private research centers.
L'archive ouverte pluridisciplinaire HAL, est destinée au dépôt et à la diffusion de documents scientifiques de niveau recherche, publiés ou non, émanant des établissements d'enseignement et de recherche français ou étrangers, des laboratoires publics ou privés. 
Muscle fascicle shortening behaviour of vastus lateralis during a maximal forcevelocity test

\section{Hugo Hauraix, Sylvain Dorel, Giuseppe Rabita, Gaël Guilhem \& Antoine Nordez}

European Journal of Applied Physiology

ISSN 1439-6319

Eur J Appl Physiol

DOI 10.1007/s00421-016-3518-4

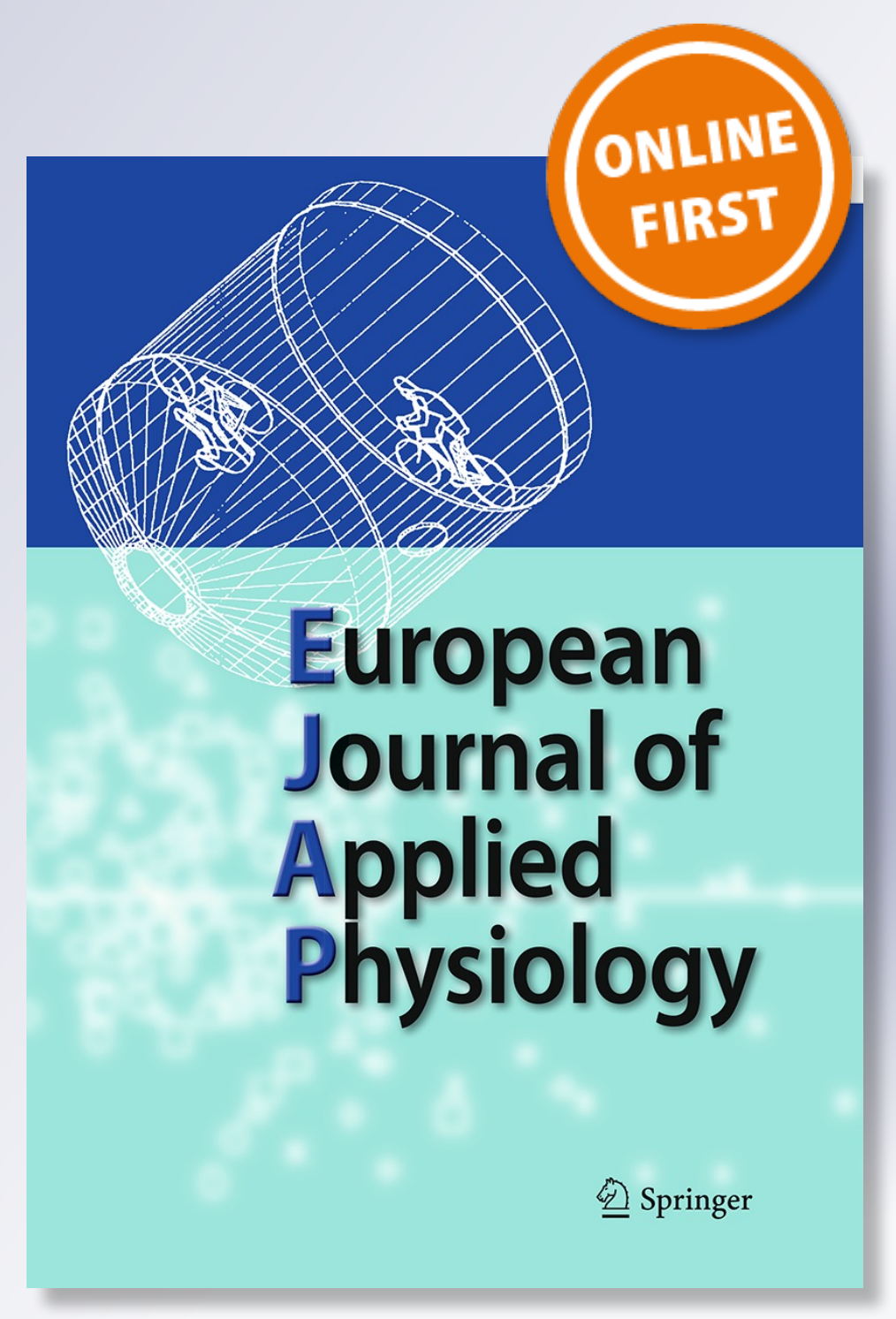

算 Springer 
Your article is protected by copyright and all rights are held exclusively by SpringerVerlag Berlin Heidelberg. This e-offprint is for personal use only and shall not be selfarchived in electronic repositories. If you wish to self-archive your article, please use the accepted manuscript version for posting on your own website. You may further deposit the accepted manuscript version in any repository, provided it is only made publicly available 12 months after official publication or later and provided acknowledgement is given to the original source of publication and a link is inserted to the published article on Springer's website. The link must be accompanied by the following text: "The final publication is available at link.springer.com". 


\title{
Muscle fascicle shortening behaviour of vastus lateralis during a maximal force-velocity test
}

\author{
Hugo Hauraix ${ }^{1} \cdot$ Sylvain Dorel $^{1} \cdot$ Giuseppe Rabita $^{2} \cdot$ Gaël Guilhem $^{2} \cdot$ Antoine Nordez $^{1}$
}

Received: 21 June 2016 / Accepted: 19 December 2016

(C) Springer-Verlag Berlin Heidelberg 2017

\begin{abstract}
Purpose Muscle fascicles-tendon interactions are the main determinant in production of high joint velocity. Currently, no study has investigated the muscle fascicles behaviour of knee extensor muscles until the highest reachable velocity (e.g., unloaded knee extension). We aimed to track the changes in vastus lateralis fascicles length during knee extensions to quantify muscle fascicles and tendinous tissues contributions to muscle-tendon unit shortening and to determine maximal muscle fascicles shortening velocity.

Methods Fifteen participants performed isokinetic and isoinertial knee extensions, and ultrafast ultrasound imaging was used to observe the vastus lateralis fascicles from low to very high joint velocity.

Results The muscle fascicles shortening velocity increased linearly with the increase in knee joint velocity up to the maximal joint velocity (mean $R^{2}=0.93 \pm 0.08$ ). Muscle fascicles contribution to muscle-tendon unit shortening velocity was almost constant regardless of the condition $(83 \pm 23 \%)$. Using Hill's equation, the maximal velocity of knee joint and muscle fascicles was determined at $1000 \pm 489^{\circ} \mathrm{s}^{-1}$ and $5.1 \pm 2.0 \mathrm{~L}_{0} \mathrm{~s}^{-1}\left(47.4 \pm 18.7 \mathrm{~cm} \mathrm{~s}^{-1}\right)$, respectively.

Conclusions Contribution of muscle fascicles to the muscle-tendon unit shortening velocity was much higher for the vastus lateralis in this study compared to the gastrocnemius
\end{abstract}

Sylvain Dorel

sylvain.dorel@univ-nantes.fr

1 Laboratory "Movement, Interactions, Performance" (EA 4334), Faculty of Sport Sciences, University of Nantes, 25 bis Boulevard Guy Mollet, BP 72206, 44322 Nantes Cedex 3, France

2 Laboratory Sport, Expertise and Performance (EA 7370), Research Department, French Institute of Sport (INSEP), Paris, France medialis in two previous studies. Moreover, this contribution of muscle fascicles shortening velocity was constant whatever the velocity condition, even at the highest reachable velocity. Thus, the vastus lateralis fascicles shortening velocity increases linearly with the knee joint velocity until high velocities and its behaviour strongly accorded with the classical Hill's force-velocity relationship.

Keywords Force-velocity relationship · Ultrafast ultrasound · Fascicles-tendon interactions - High velocity · Knee extension
Abbreviations
ANOVA Analysis of variance
$L_{0} \quad$ Optimal length

\section{Introduction}

Mechanical interactions between muscle fascicles and tendinous tissues (i.e., tendon and aponeurosis) contribute to efficient movements (Ettema et al. 1990). These interactions have been well described in animal (Astley and Roberts 2012; Biewener and Baudinette 1995; Wilson et al. 2003) and human locomotion (e.g., walking and running; Cronin and Lichtwark 2013; Cronin et al. 2013; Fukunaga et al. 2001; Ishikawa et al. 2007; Lichtwark et al. 2007). During exercises involving stretch-shortening cycles, these interactions have been demonstrated to enhance the work produced by the muscle-tendon unit (Ishikawa and Komi 2004; Kawakami et al. 2002; Finni et al. 2001), to improve the efficiency (Lichtwark and Barclay 2010) and to reduce muscle fibre elongation during the eccentric phase, thereby limiting the risk of strain induced injury (Guilhem et al. 2016; Konow et al. 2012). 
Muscle fascicles-tendon interactions are also involved in concentric contractions of the knee extensors (De Brito Fontana et al. 2014; Finni et al. 2003; Ichinose et al. 2000) and plantar flexors (Chino et al. 2008; Hauraix et al. 2013, 2015; Randhawa et al. 2013). For instance, during a maximal plantar flexion, the initial ankle dorsiflexion position induces stretching of the muscle-tendon unit and hence of the tendinous tissues before the contraction (Hauraix et al. 2013). Subsequently, when the contraction starts and after a possible additional slight lengthening of tendinous tissues induced by shortening of muscle fascicles, the elastic tendinous tissues are, in turn, shortened, contributing significantly to the shortening velocity of the muscle-tendon unit (up to $46 \%$ ). Interestingly, during these maximal concentric contractions, the respective contributions of muscle fascicles and tendinous tissues to muscle-tendon unit shortening velocity did not change with the movement velocity from slow plantar flexions $\left(30^{\circ} \mathrm{s}^{-1}\right)$ up to maximal joint velocity (Hauraix et al. 2013, 2015).

To our knowledge, among the previous studies, only one investigated muscle fascicles-tendon interactions during knee extensions performed with a large range of velocities (i.e., up to $500^{\circ} \mathrm{s}^{-1}$, De Brito Fontana et al. 2014). Contrary to the results obtained for plantar flexions, according to these authors, the muscle fascicle shortening velocity plateaued for joint velocities above $240^{\circ} \mathrm{s}^{-1}$, suggesting an increase in the tendinous tissues contribution with the increase in knee extension velocity (up to $90 \%$ at $500^{\circ} \mathrm{s}^{-1}$ ). The discrepancy with the results obtained by Hauraix et al. $(2013,2015)$ on plantar flexor muscles could reflect different muscle fascicles-tendon interactions, according to the joint tested (ankle vs. knee), which may be associated with different mechanical tendon properties. In addition, De Brito Fontana et al. (2014) measured the mean muscle fascicle shortening velocity during the entire motion, including acceleration as well as isokinetic and deceleration phases, while Hauraix et al. $(2013,2015)$ continuously tracked muscle fascicle during the movement to appraise the "instantaneous" muscle fascicle shortening velocity. Considering that (1) the respective contributions of muscle fascicles and tendinous tissues are highly dependent on the range of motion considered during the movement (Hauraix et al. 2013), and the fact that (2) ultrasound data were sampled at very low frequencies $(42-49 \mathrm{~Hz}$, which is not recommended in the study of fast movements, Cronin and Lichtwark 2013), the chosen methodological approach may strongly influence the subsequent results and potentially explain the observed differences. Finally, the range of joint velocities investigated in the study of De Brito Fontana et al. (2014) (i.e., up to $500^{\circ} \mathrm{s}^{-1}$ ) remained very far from the maximal velocity that the joint is able to reach during a single-joint concentric contraction (between 700 and $1500^{\circ} \mathrm{s}^{-1}$; Forrester et al. 2011; Tihanyi et al. 1982; Thorstensson et al. 1977). Then, the reported stagnation of the muscle fascicle shortening velocity remains surprising, because that would mean that these very fast knee extensions from $400^{\circ} \mathrm{s}^{-1}$ up to this maximal joint velocity would be almost exclusively produced by the involvement of tendinous tissues. As stated, it would interfere with the interpretation of the torque-angular velocity relationship and, more specifically, the link classically made between the maximal angular velocity (determined from the Hill's modelling relationship) and the maximal shortening velocity of muscle fibres. Consequently, it would be interesting to explore the muscle fascicle behaviour during the highest knee joint velocities achieved by humans (e.g., during a kick) to investigate whether, in the same way as for as the plantar flexors, a classical Hill's force-velocity relationship would also be obtained at the fascicle level for the knee extensors.

The current study was designed to investigate muscle fascicles behaviour during maximal knee extensions performed at a large range of joint velocities (i.e., force-velocity test): from low $\left(100^{\circ} \mathrm{s}^{-1}\right)$ to the maximal achievable velocity. To this end, an ultrafast ultrasound with high sampling frequency (i.e., until $2000 \mathrm{~Hz}$ ) was used to track the instantaneous muscle fascicle length changes during very fast unloaded movements. The purpose was to quantify muscle fascicle and tendinous tissues contributions to muscle-tendon unit shortening at each velocity and to describe the nature of the relationship between muscle fascicle shortening velocity and joint velocity. Additional aim was to establish the torque-velocity relationship and the forcevelocity relationship of vastus lateralis fascicles and hence to determine the knee extensor maximal shortening velocity in vivo, providing the first data for future comparative studies.

\section{Materials and methods}

\section{Participants}

Fifteen healthy men $(22.8 \pm 3.2$ years, $179.0 \pm 5.5 \mathrm{~cm}$, $75.4 \pm 6.2 \mathrm{~kg}$ ) volunteered to participate in this study. All participants were engaged in physical activity, ranging from recreational to high-level competition (i.e., sprint running, basketball, soccer, gymnastics, rowing, handball, power lifting, dance, and tennis). Subjects were fully informed about the nature and aim of the protocol, before signing a written informed consent form indicating their willingness to participate in the study. This study conformed to the Declaration of Helsinki. 

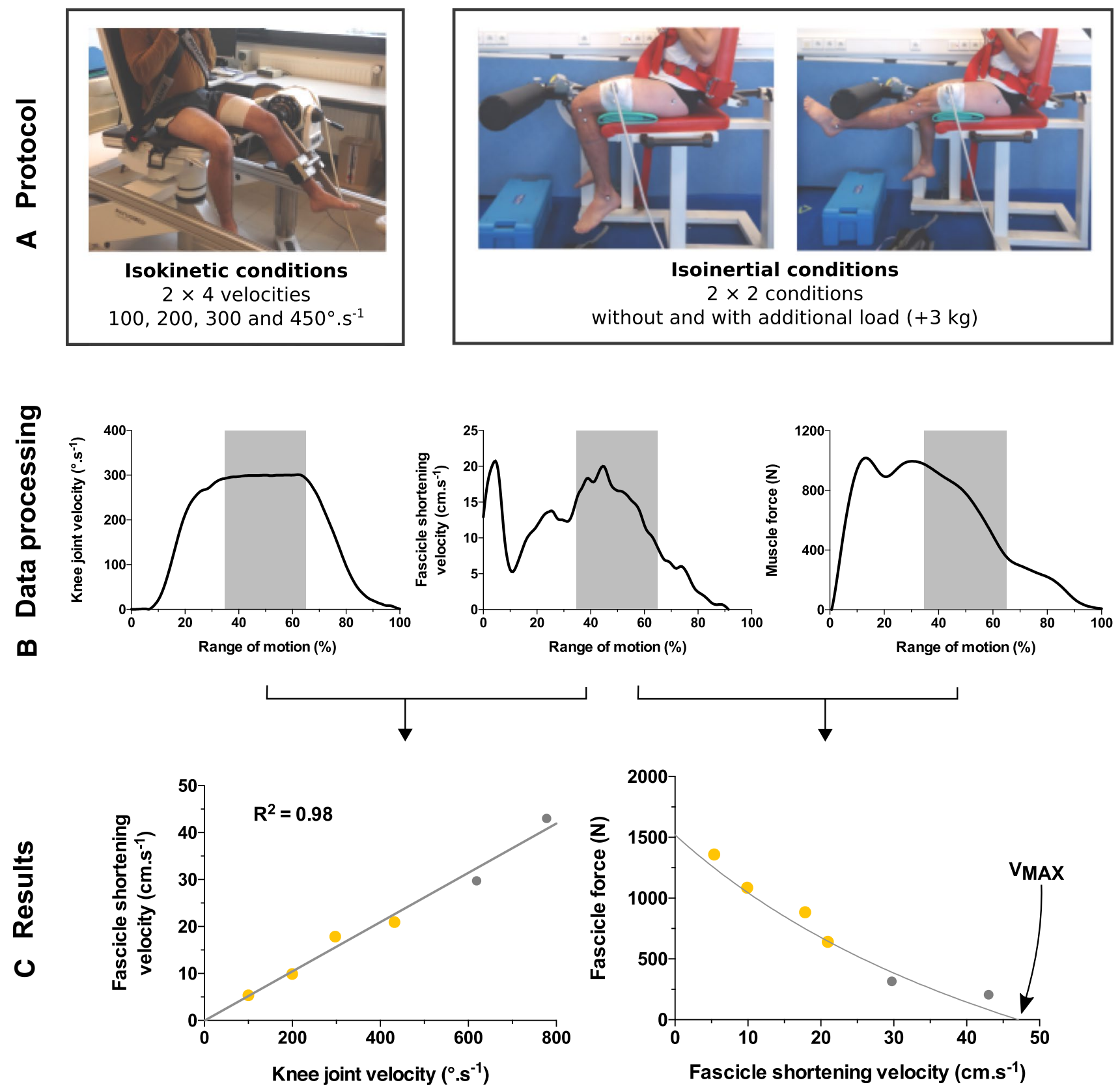

Fig. 1 Individual example of data processing and obtained results. Participants performed two trials at four isokinetic velocities and two isoinertial conditions (a). The joint velocity, muscle fascicle shortening velocity, and muscle force were averaged over an $80^{\circ}-50^{\circ}$ range of motion (b, grey-coloured area). For each participant, the muscle

fascicle shortening-joint velocity relationship and force-velocity relationship of muscle fascicle were fitted using linear and hyperbolic (Hill) model, respectively (c). Yellow and gray points corresponded to isokinetic and isoinertial conditions, respectively

\section{Mechanical measurements}

Knee extensions were performed on an isokinetic dynamometer (Con-Trex, CMV AG, Dübendorf, Switzerland). Joint angle, velocity, and torque were digitized using a 12-bit analogue to digital converter (PowerLab 16/35, AD Instruments, Australia) at $1000 \mathrm{~Hz}$. The participants were seated with the hip joint flexed at $80^{\circ}\left(0^{\circ}\right.$ correspond to the

hip fully extended; Fig. 1a). Their shoulders and hips were secured by adjustable lap belts and held in position. The input axis of the dynamometer was carefully adjusted to the knee's axis of rotation.

High-velocity knee extensions were performed on an adapted bench (Fig. 1a). The contractions were stopped by a damping system to assist the end of the movement and to limit a potential inhibition of agonist muscles. A 
three-camera motion analysis system (Vicon Motion System Ltd., Oxford, UK) recorded the three-dimensional coordinates of reflective markers located on the following body landmarks of the left leg: lateral malleolus, tibia head, lateral femoral epicondyle, and the largest protuberance of the greater trochanter (Fig. 1). The sampling frequency was set to $250 \mathrm{~Hz}$.

\section{Ultrasound measurements}

An ultrafast ultrasound device was used to observe the fascicles of vastus lateralis muscle during knee extensions (Aixplorer, Supersonic Imagine, Aix en Provence, France). The probe (5-12 MHz, $55 \mathrm{~mm}$ ) was placed on the skin surface over the muscle belly at $39 \%$ of the distance between the greater trochanter and the lateral femoral epicondyle (Blazevich et al. 2006). The orientation of the probe followed the recommendations of Benard et al. (2009) to obtain the longest muscle fascicles possible. A custommade equipment was used to securely maintain the probe on the left leg. According to pilot studies, ultrasound sampling frequency was set between 500 and $2000 \mathrm{~Hz}$, depending on the movement velocity, to allow an accurate muscle fascicle tracking with a good temporal resolution (see below). Ultrasound measurements were synchronized with mechanical data and kinematic data using an external trigger of the ultrasound scanner sampling using a 16-bit A/D converter (PowerLab 16/35, AD Instruments, Australia).

\section{Protocol}

After a standardized warm-up, subjects performed two trials of maximal knee extension at four isokinetic velocities $\left(100,200,300\right.$, and $\left.450^{\circ} \mathrm{s}^{-1}\right)$ in a randomized order, with 2 min rest between each trial (Fig. 1a). The starting angle position of knee joint was set at $110^{\circ}$, and the participants performed knee extension until $10^{\circ}$ (i.e., where $0^{\circ}$ corresponds to the full extension). After each extension, the knee was passively placed in starting position. The previous studies showed higher maximal joint velocity during unloaded plantar flexions performed with preactivation (1325 $\mathrm{s}^{-1}$, Farcy et al. 2014) compared to without pre-activation $\left(701^{\circ} \mathrm{s}^{-1}\right.$, Hauraix et al. 2015). However, the maximal fascicle shortening velocity was much higher without pre-activation $\left(23.2 \mathrm{~cm} \mathrm{~s}^{-1}\right.$ compared to $34.7 \mathrm{~cm} \mathrm{~s}^{-1}$ ). These findings highlighted the importance of pre-activation on muscle-tendon interactions. Since the aim was to reach the highest fascicle shortening velocities as possible in a concentric mode, and hence limit the contribution of elastic properties, subjects were asked to be fully relaxed before the contraction (i.e., without pre-activation). After a brief countdown, the participant performed a maximal knee extension throughout the range of motion.
In our experience, the automatic tracking was much more accurate when the difference between successive images is small and hence when the temporal resolution (or frame rate) increases. Because the ultrafast ultrasound scanner used in this study did not permit to record more than 1000 frames per acquisition, the sampling frequency was set to cover the entire motion with an appropriate temporal resolution to track the muscle fascicle easily (see below). Thus, ultrasound sampling frequency was set at $500 \mathrm{~Hz}$ for $100^{\circ} \mathrm{s}^{-1}$ and $1000 \mathrm{~Hz}$ for the other isokinetic conditions, and $2000 \mathrm{~Hz}$ for isoinertial conditions. Subsequently, participants performed a series of maximal isoinertial knee extensions on the adapted bench. The body position and the range of motion were the same as in isokinetic conditions. Two conditions were tested (two trials per condition), with and without an additional load applied using a $3 \mathrm{~kg}$-sandbag fixed on the subjects' ankle. An ultrasound acquisition was performed at each trial $(2000 \mathrm{~Hz})$. The motion analysis system was used to calculate the knee joint angle during isoinertial knee extensions.

\section{Data processing}

Data processing was performed using custom-written MATLAB scripts (The MathWorks, Natick, MA). The joint angle, velocity, and torque were low-pass filtered $(20 \mathrm{~Hz})$ using a zero-phase second-order Butterworth filter for isokinetic conditions. The external torque produced during isokinetic knee extension was corrected using acceleration to remove the inertia effects of leg and dynamometer equipment. After that, the weight of leg and dynamometer equipment was taken into consideration to obtain the external torque at the knee joint. The reflective markers' positions obtained during isoinertial conditions were filtered using a second-order low-pass Butterworth filter with a cut-off frequency of $16 \mathrm{~Hz}$ (Bobbert et al. 1986). In this condition, knee joint angle was calculated in the sagittal plan from markers' positions and joint velocity was obtained by finite differences. The external torque was calculated from the joint acceleration, the moments of inertia, and the weights of the leg, the foot, and the potential additional load. The external torque due to inertia effects was equal to the knee joint acceleration multiplied by the sum of all moments of inertia in the system (i.e., foot, leg, and additional load). The moment of inertia of foot and leg was calculated with respect to the rotation axis of the knee using parallel axis theorem proposed by de Leva (1996). The inertia of the additional load was calculated as the load of the sandbag ( $3 \mathrm{~kg}$ ) multiplied by the squared radius of gyration, which corresponds to the distance between the rotation axis of knee and the centre of sandbag.

The individual lengths of the muscle-tendon unit and moment arm were obtained using anthropometric model 
and the knee joint angle (Visser et al. 1990). B-mode images were created from ultrasonic raw data using a conventional beam formation (i.e., applying a time delay operation to compensate for travel time differences). The displacements of muscle fascicle, deep, and superficial aponeurosis were measured using the automatic tracking method proposed by Cronin et al. (2011). Vastus lateralis fascicles were not always fully visible on B-Mode images. Therefore, the muscle fascicle length was extrapolated using trigonometry method assuming linear muscle fascicles and aponeurosis (Finni et al. 2003; Hauraix et al. 2013). Pennation angle was calculated as the angle formed between deep aponeurosis and muscle fascicle. Muscle fascicle length and pennation angle were low-pass filtered by a zero-phase second-order Butterworth filter with an adapted cut-off frequency according to the ultrasound sampling frequency (cut-off frequency of 15,20 , and $40 \mathrm{~Hz}$, for 500,1000 , and $2000 \mathrm{~Hz}$ of sampling frequency, respectively). Horizontal projection of muscle fascicle length was calculated as the muscle fascicle length multiplied by the cosine of pennation angle. The tendinous tissues length was calculated as the muscle-tendon unit length minus the horizontal projection of muscle fascicle length (Fukunaga et al. 2001). The shortening velocities of muscle-tendon unit, muscle fascicle, and tendinous tissues were obtained by a time derivative of the changes in their respective length. To estimate the muscle fascicles contribution to the muscle-tendon unit shortening velocity, the horizontal muscle fascicle shortening velocity was divided by the shortening velocity of muscle-tendon unit. The muscle fascicle shortening velocity was also expressed in relative value and then reported in optimal length per second $\left(\mathrm{L}_{0} \mathrm{~s}^{-1}\right)$, considering a mean optimal length estimated to $9.3 \mathrm{~cm}$ according to Austin et al. (2010).

Knee extensors force was calculated as the external torque divided by the specific angle moment arm (Ichinose et al. 2000). Vastus lateralis force was estimated as the knee extensors force multiplied by the relative volume of vastus lateralis to the global volume of the knee extensor muscles (34\%, Akima et al. 1995). Considering that muscle fascicles are arranged in parallel, the force produced by all muscle fascicles was calculated as the vastus lateralis force divided by the cosine of the pennation angle (Fukunaga et al. 1996). The analysis focused on the $80^{\circ}-50^{\circ}$ range of motion for all conditions (Fig. 1b). Joint velocity, torque, muscle-tendon unit shortening velocity, muscle fascicle shortening velocity, and muscle fascicles force were averaged over this range of motion and used to build joint velocity-muscle fascicle shortening velocity relationship, the torque-velocity relationship, and the force-velocity relationship of muscle fascicle (Fig. 1c). The model for the Eq. 1 was fitted using the linearization method proposed by Hill (1938),
$(F+a)(V+b)=c$,

where $F$ and $V$ correspond to the force (or torque) and velocity of muscle fascicle (or knee joint), respectively, and $a, b$, and $c$ are constants. The intercept of $V$-axis corresponds to the maximal velocity.

\section{Statistical analysis}

The normality of distribution was checked using ShapiroWilk's test. Since all data were normally distributed, the statistical changes in muscle fascicle shortening velocity and the relative contributions to muscle-tendon unit shortening according to the six velocity conditions (i.e., four isokinetic conditions and two isoinertial conditions) were evaluated using a one-way ANOVA. When the sphericity assumption was violated (Mauchly test), a Geisser-Greenhouse correction was used. A Newman-Keuls post hoc analysis was conducted when appropriate. A linear fit was performed to examine the relation between the muscle fascicle shortening velocity and the joint velocity for each participant. The corresponding coefficient of determination was used to evaluate the linearity of this relationship. Hill's hyperbolic model was fitted to the torque-velocity relationship, and muscle fascicle force-velocity relationship. The fitting of the model was measured by the coefficient of determination $\left(R^{2}\right)$. The student's paired $t$ test and the Bravais-Pearson correlation coefficient $(r)$ were used to evaluate the difference and relationship between the muscle fascicle shortening velocity measured during isoinertial without an additional load and the maximal velocity extrapolated. The data are presented as mean $\pm \mathrm{SD}$. The level of significance was set as $p<0.05$.

\section{Results}

The patterns of instantaneous joint velocity, muscle-tendon unit shortening velocity, muscle fascicle shortening velocity, horizontal muscle fascicle shortening velocity, and tendinous tissues shortening velocity are presented in Fig. 2 for all tested conditions. As expected, isoinertial conditions elicited the highest joint velocity (Fig. 2a). In these conditions, the muscle fascicle shortening velocity (Fig. 2c) increased during knee extension up to a joint angle of $45^{\circ}$ and then dropped towards the end of the movement. The behaviour of tendinous tissues was divided into two phases for all tested conditions. During the first part of the movement, tendinous tissues were stretched until approximately $75^{\circ}-80^{\circ}$ of knee joint angle. Subsequently, tendinous tissues shortened up to the end of the movement.

The mean shortening velocity of muscle fascicles reached $34.6 \pm 12.4 \mathrm{~cm} \mathrm{~s}^{-1}$ for the isoinertial condition without an additional load, while mean joint velocity 
Fig. 2 Joint velocity (a), muscle-tendon unit shortening velocity (b), muscle fascicle shortening velocity (c), horizontal muscle fascicle shortening velocity (d), and tendinous tissues shortening velocity (e) patterns measured during maximal knee extensions at various isokinetic pre-set velocities and isoinertial knee extension with and without an additional load. Patterns were averaged across participants. The range of motion used for further analysis is represented by the black frame $\left(80^{\circ}-50^{\circ}\right)$. Error bars were omitted for clarity
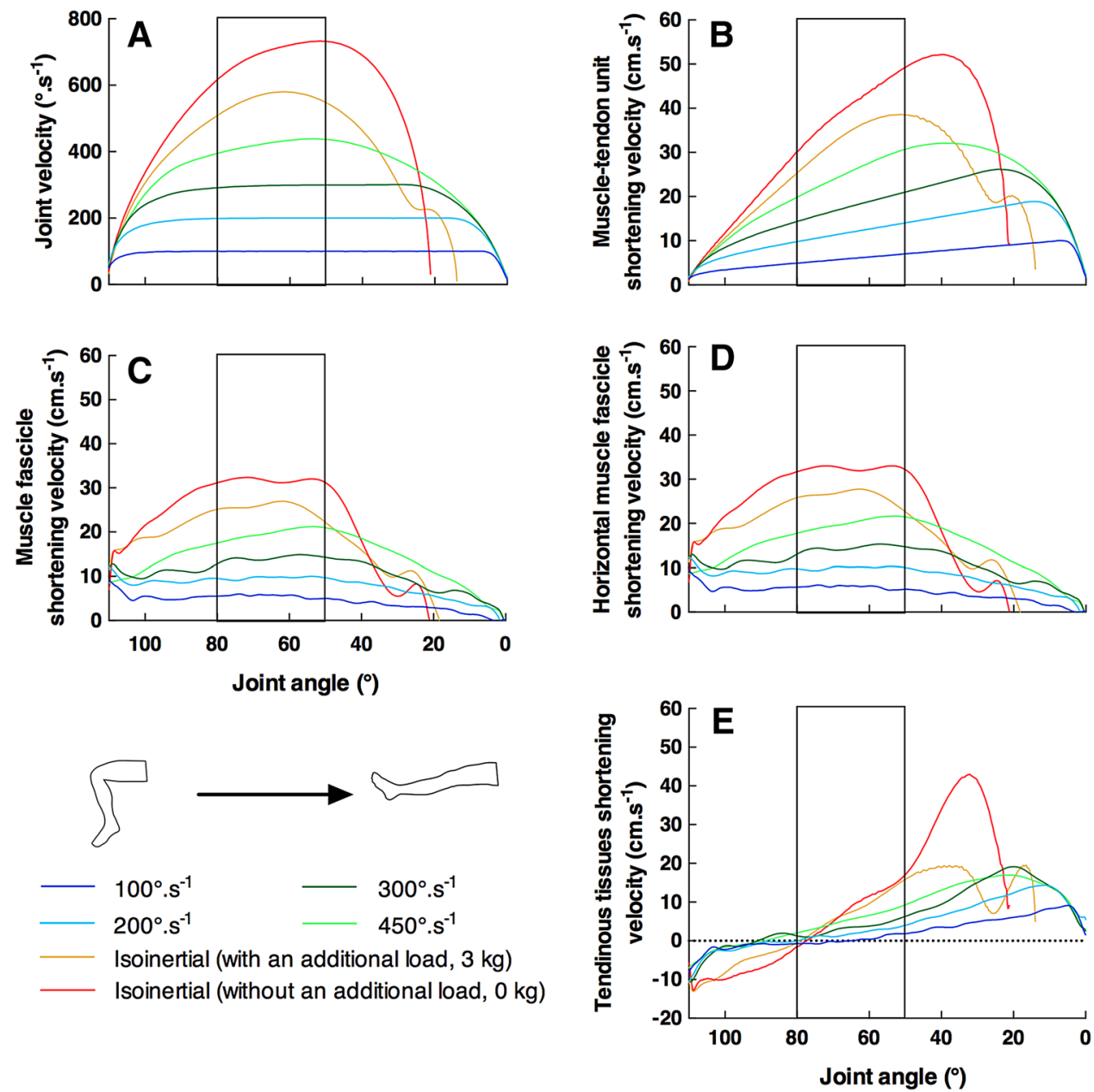

Table 1 Knee joint velocity $\left(V_{\mathrm{K}}\right)$, muscle fascicle shortening velocity $\left(V_{\mathrm{F}}\right)$, and the muscle fascicles contribution to muscle-tendon unit shortening velocity $\left(C_{\mathrm{F}}\right)$ for each velocity condition and the maximal shortening velocity extrapolated from the Hill's modelling forcevelocity relationship $\left(V_{\mathrm{Max}}\right)$

\begin{tabular}{lcclllll}
\hline & $100^{\circ} \mathrm{s}^{-1}$ & $200^{\circ} \mathrm{s}^{-1}$ & $300^{\circ} \mathrm{s}^{-1}$ & $450^{\circ} \mathrm{s}^{-1}$ & Isoinertial load & Isoinertial no load & $V_{\mathrm{Max}}$ \\
\hline$V_{\mathrm{K}},{ }^{\circ} \mathrm{s}^{-1}$ & $100(0)$ & $200(0)^{* * *}$ & $297(1)^{* * *}$ & $432(1)^{* * *}$ & $610(81)^{* * *}$ & $750(82)^{* * *}$ \\
$V_{\mathrm{F}}, \mathrm{cm} \mathrm{s}^{-1}$ & $5.6(0.9)$ & $9.7(1.6)^{* * *}$ & $14.2(2.7)^{* * *}$ & $19.8(4.7)^{* * *}$ & $28.1(12.2)^{* * *}$ & $34.5(12.4)^{* * *}$ & $1000(489)$ \\
$\mathrm{C}_{\mathrm{F}}, \%$ & $96.9(20.8)$ & $84.9(18.4)^{* *}$ & $82.2(17.1)$ & $78.3(20.2)$ & $80.6(32.0)$ & $80.8(26.3)$ & $4(18.7)^{* * * \dagger}$ \\
\hline
\end{tabular}

Data are the mean (SD)

Significantly different with the slower velocity condition $(* * * p<0.001, * * p<0.01)$

Significantly correlated with the isoinertial no-load condition $\left({ }^{\dagger} p<0.05\right)$

reached $750 \pm 82^{\circ} \mathrm{s}^{-1}$ on average (mean values were calculated from $80^{\circ}$ to $50^{\circ}$ ). All mean values and statistical changes are presented in Table 1.

We observed that muscle fascicles contribution to the shortening velocity of muscle-tendon unit decreases as knee joint velocity increased (velocity effect $p=0.051$ ). However, the muscle fascicles contributions between velocity conditions were similar (ranging from 78.3 to $84.9 \%$, Fig. 3), except for the slowest condition (i.e., $30^{\circ} \mathrm{s}^{-1}, 96.9 \%$,
Table 1). A significant main effect of condition $(p<0.001)$ indicated a significant increase in muscle fascicle velocity of vastus lateralis, while knee joint velocity increased (Fig. 3). Whatever the condition tested, the muscle fascicle shortening velocity and the knee joint velocity were significantly higher than those observed at the slower and closest velocity condition (Table 1). Individual relationships between muscle fascicle velocity and joint velocity were well fitted by the linear model (Fig. 4, mean $R^{2} 0.93 \pm 0.08$, range 0.68-0.99). 


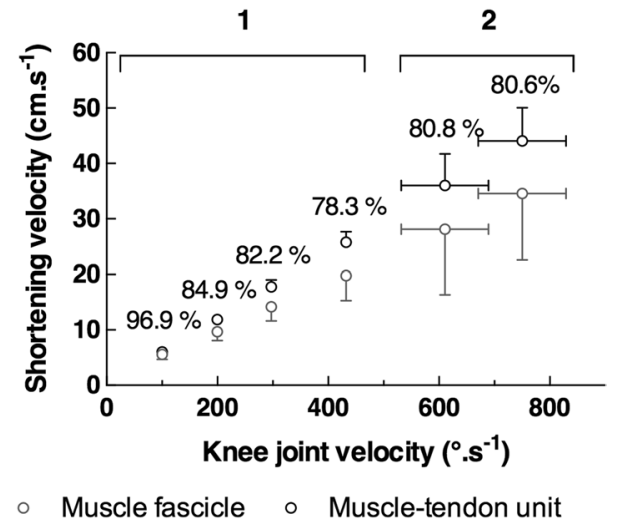

Fig. 3 Mean \pm SD horizontal muscle fascicle velocity and muscle-tendon unit velocity are presented for each condition (four isokinetic conditions: 1; two isoinertial conditions: 2). Mean relative contributions of muscle fascicles to muscle-tendon unit velocity are expressed as percentages

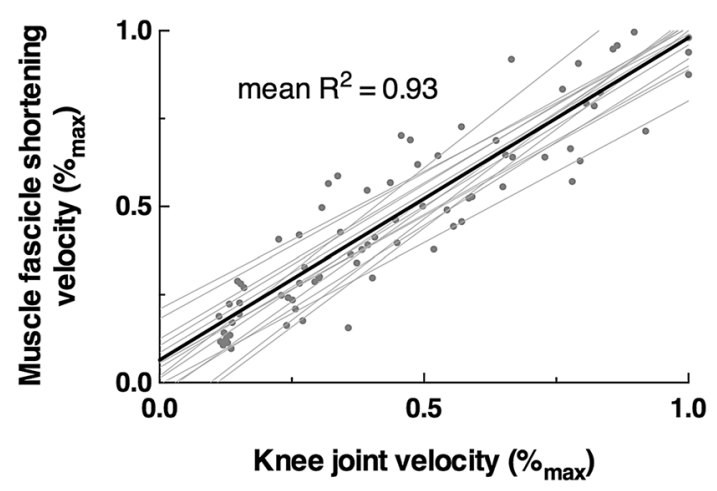

Fig. 4 Muscle fascicle velocity-joint velocity relationship for all trials. Muscle fascicle velocity and joint velocity in each condition were normalized to maximal values obtained during isoinertial condition without an additional load. Gray lines (individual models) and black line (mean trend curve $n=15$ ) are shown for clarity purposes

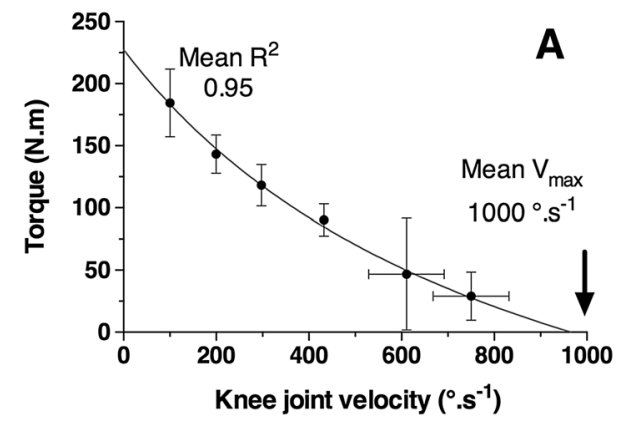

Fig. 5 Torque-velocity (a) and muscle fascicles force-velocity relationships (b) based on mean values of all subjects over the analysed range of motion $\left(80^{\circ}-50^{\circ}\right)$. Each individual force-velocity curve was
For each participant, individual torque-velocity and muscle fascicle force-velocity relationships were well fitted by the hyperbolic model proposed by Hill (Fig. 5, mean $R^{2} 0.95 \pm 0.05$ and mean $R^{2} 0.91 \pm 0.08$, respectively). The maximal joint velocity extrapolated by Hill's equation reached $1000 \pm 489^{\circ} \mathrm{s}^{-1}$ on average and ranged from 665 to $2426^{\circ} \mathrm{s}^{-1}$. The maximal muscle fascicle shortening velocity was $47.4 \pm 18.7 \mathrm{~cm} \mathrm{~s}^{-1}$ or $5.1 \pm 2.0 \mathrm{~L}_{0} \mathrm{~s}^{-1}$ on average. These values were significantly higher compared to muscle fascicle shortening velocity assessed during isoinertial conditions $\left(34.6 \pm 12.0 \mathrm{~cm} \mathrm{~s}^{-1} ; p<0.0001\right)$. The maximal muscle fascicle shortening velocity and the muscle fascicle shortening velocity measured during isoinertial condition without an additional load were positively correlated $(r=0.63, p=0.02)$.

\section{Discussion}

This study showed that the muscle fascicles shortening velocity continuously increases as a function of the increase in knee joint velocity. In addition, a linear model captured well the relationship between both variables. The respective contributions of muscle fascicles and tendinous tissues to the muscle-tendon unit shortening velocity were almost constant, regardless of the condition tested. Using Hill's equation, maximal knee joint velocity and muscle fascicles shortening velocity of vastus lateralis muscle were estimated on average at $1000 \pm 489^{\circ} \mathrm{s}^{-1}$ and $5.1 \pm 2.0 \mathrm{~L}_{0} \mathrm{~s}^{-1}$, respectively.

\section{Muscle fascicles and tendinous tissues behaviours during concentric knee extension}

This study is the first to track the instantaneous length changes in vastus lateralis muscle-tendon unit, muscle fascicle, and tendinous tissues during knee extensions up to

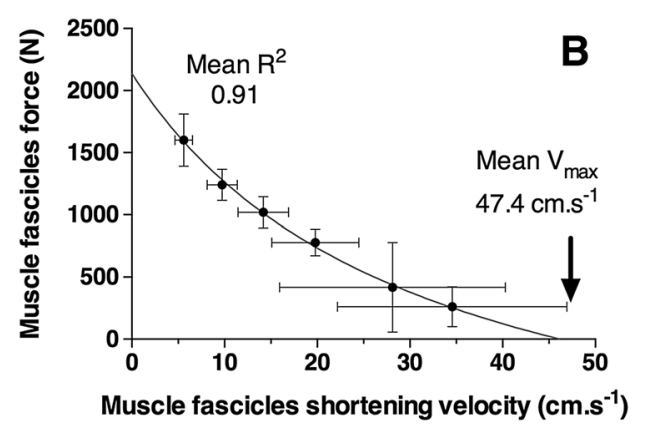

fitted by the hyperbolic equation proposed by Hill (1938). The mean of individual $R^{2}$ and maximal velocity are shown and correspond to the values obtained from all subjects $(n=15)$ 
the highest reachable velocity. As previously reported in the literature on various muscles, the muscle fascicle behaviour (length changes and shortening velocity) differed from the behaviour of muscle-tendon unit during concentric contraction (De Brito Fontana et al. 2014; Hauraix et al. 2013, 2015; Ichinose et al. 2000; Randhawa et al. 2013). Indeed, the shortening velocity of muscle fascicle was always lower compared to the muscle-tendon unit shortening velocity, and the shape of both curves over the range of motion clearly differed (Fig. 2b, c). This phenomenon is classically explained by the interactions between muscle fascicles and tendinous tissues (Ichinose et al. 2000). Moreover, the starting knee angle $\left(110^{\circ}\right)$ induced an initial pre-stretching of tendinous tissues, as previously observed during plantar flexion (Hauraix et al. 2015). Interestingly, as depicted in Fig. 2e, tendinous tissues were additionally significantly stretched in the first $25^{\circ}$ of the range of motion, regardless of the velocity condition tested, which may be considered as a storage of elastic energy. Subsequently, despite the "concentric" modality of exercise, an elastic energy was released, resulting in a shortening of tendinous tissues throughout the lasting range of motion. As reflected by the tendinous tissues shortening velocity patterns (Fig. 2e), the storage of elastic energy was more important under isoinertial loading, allowing for high elastic energy restitution. Thus, the muscle-tendon unit can reach high shortening velocities thanks to these specific muscle fascicles-tendon interactions (Hauraix et al. 2015).

\section{Relationship between muscle fascicles shortening velocity and joint velocity}

Our results showed that the muscle fascicles shortening velocity increased linearly with the increase in knee joint velocity (Figs. 3, $4, R^{2}=0.93$ ). In other words, the contribution of muscle fascicles to muscle-tendon unit shortening velocity was almost constant up to the highest knee joint velocities that the subjects were able to reach (Fig. 3). Only one study on the muscle fascicles behaviour at high joint velocity $\left(500^{\circ} \mathrm{s}^{-1}\right)$ highlighted that the muscle fascicles shortening velocity reaches a plateau at joint velocities above $240^{\circ} \mathrm{s}^{-1}$ (De Brito Fontana et al. 2014). Our results are inconsistent with this study, and methodological considerations could explain this discrepancy. De Brito Fontana et al. (2014) measured an average shortening velocity of muscle fascicles during three different phases of isokinetic movements (acceleration, isokinetic, and deceleration phases). Due to the inertia of the ergometer and the time required to achieve the pre-set velocity, the relative time of isokinetic phase declines with the increase of preset isokinetic velocity (95-20\% for 45 and $500^{\circ} \mathrm{s}^{-1}$, respectively, De Brito Fontana et al. 2014; Osternig 1986). However, the instantaneous tracking of muscle fascicle performed in this study clearly shows that the mean and maximal instantaneous shortening velocities of muscle fascicle continue to increase according to the velocity condition (Fig. 2c). Moreover, the sampling frequency offered by the ultrafast ultrasound scanner (ranged between 500 and $2000 \mathrm{~Hz}$ ) is higher compared to those offered by standard ultrasound scanners used in the aforementioned study $(42-49 \mathrm{~Hz})$. Since the instantaneous variations in muscle fascicle length are important, it seems essential to sample them at high frame rates. For example, at a joint isokinetic velocity of $240^{\circ} \mathrm{s}^{-1}$ with a $40^{\circ}$ of range of analysis, a standard ultrasound device records approximately 8-9 frames (for sampling frequency at $50 \mathrm{~Hz}$ ), while an ultrafast ultrasound records 167 frames (for sampling frequency at $1000 \mathrm{~Hz}$ ). Specifically, it has been demonstrated that a low sampling frequency limits the accuracy with which the muscle fascicle length changes can be observed during dynamic contractions above $200^{\circ} \mathrm{s}^{-1}$ (Cronin and Lichtwark 2013). Currently, ultrafast ultrasound is the only device that allows studying the behaviour of the different components of muscle-tendon unit during high-velocity contractions.

The constant contribution of muscle fascicles to muscle-tendon unit shortening is in agreement with the one observed for the gastrocnemius medialis muscle of ankle joint (Hauraix et al. 2015). However, we observed that the muscle fascicles average contribution to muscle-tendon unit shortening is lower during plantar flexion than during knee extension $(60 \pm 14$ and $83 \pm 23 \%$, respectively). Although the main purpose of the study of Kubo et al. (2014) was not directly related to the topic of the current work, the authors observed a large difference of tendon properties between knee extensors and plantar flexors muscles. This result highlights the different contribution of patellar and Achilles tendinous tissues to muscle-tendon unit shortening in relation with their respective mechanical properties. More precisely, the higher stiffness of vastus lateralis tendinous tissues could explain that the muscle fascicles have to produce superior relative shortening velocity for the vastus lateralis muscle compared to the gastrocnemius medialis muscle. Moreover, considering the ratio between the rest length of tendinous tissues and the rest length of muscle-tendon unit, we observed a significant difference between vastus lateralis $(75.6 \%)$ and gastrocnemius medialis ( $86.5 \%$, Hauraix et al. 2015). This ratio can also partly explain the larger participations of vastus lateralis fascicles to the muscle-tendon unit shortening velocity. Consequently, it is not surprising to observe a larger muscle fascicles contribution to muscle-tendon unit shortening velocity for the knee extension than for plantar flexion.

\section{Estimation of maximal knee joint velocity and muscle fascicles shortening velocity of vastus lateralis}

Few studies have attempted to estimate the maximal knee joint extension velocity using an extrapolation method 
permitted by the use of Hill's equation (Forrester et al. 2011; Tihanyi et al. 1982). By combining measurements under isokinetic and isoinertial resistances, these authors have reported individual data of maximal knee joint velocity, ranging from 680 to $1610^{\circ} \mathrm{s}^{-1}$. Our results of extrapolated maximal joint velocities are in accordance with these previous studies $\left(1000 \pm 489^{\circ} \mathrm{s}^{-1}\right.$ on average, ranged between 665 and $2426^{\circ} \mathrm{s}^{-1}$ ) and highlight the importance of performing trials using low isoinertial resistances to estimate a maximal velocity.

The highest velocity measured during our protocol (i.e., isoinertial condition without additional load) was significantly lower compared to the maximal extrapolated velocity $\left(750 \pm 82^{\circ} \mathrm{s}^{-1}\right.$, i.e., approximately $75 \%$ of the maximal extrapolated velocity). This finding can easily be explained by the effect of the weight of the participant's leg. Indeed, the mean torque produced during an isoinertial condition without additional load, which was due to both inertial and gravity effects, reached about $12 \%$ of the maximal voluntary contraction (averaged on analysis range $80-50^{\circ}$ ). Accordingly, the muscle fascicle shortening velocity measured during isoinertial condition without additional load was also significantly lower compared to the extrapolated value $\left(p<0.0001 ; 34.6 \pm 12.0\right.$ and $47.4 \pm 18.7 \mathrm{~cm} \mathrm{~s}^{-1}$, respectively). The significant correlation between the estimated maximal shortening velocity and the shortening velocity obtained during the isoinertial condition was relatively poor $(r=0.63, p=0.02)$. Overall, this result demonstrates that contrary to the gastrocnemius medialis muscle of the ankle joint (Hauraix et al. 2015), it is not appropriate to use only one isoinertial contraction performed without additional load to determine a representative maximal joint or muscle fascicle velocity (at least with this protocol, which does not offset the effects of inertia and weight of the leg).

To the best of our knowledge, no study has previously accounted for the muscle fascicles shortening velocity during this type of very fast isoinertial knee extensions. Maximal muscle fascicles shortening velocity reached $47.4 \pm 18.7 \mathrm{~cm} \mathrm{~s}^{-1}$ on average. Note that this value is higher compared to the maximal muscle fascicles shortening velocity of gastrocnemius medialis muscle during isoinertial plantar flexion $\left(30.8 \pm 5.8 \mathrm{~cm} \mathrm{~s}^{-1}\right.$, Hauraix et al. 2015). However, when normalized to the optimal fibre length (i.e., in $\mathrm{L}_{0} \mathrm{~s}^{-1}$, with $L_{0}=9.3 \mathrm{~cm}$, Austin et al. 2010), both maximal shortening velocities were similar $(5.1 \pm 2.2$ and $6.2 \pm 1.2 \mathrm{~L}_{0} \mathrm{~s}^{-1}$ for VL and GM, respectively). The maximal shortening velocity of fascicle depends on the length of fascicle (here cancelled out by the normalization; Wickiewicz et al. 1984) and the proportion of fast-twitch fibres (Barany 1967). Thus, considering the almost similar proportion of fast-twitch fibres in both muscles (Johnson et al. 1973), our results are consistent.

\section{Methodological considerations}

Although the musculoskeletal model used in this study is commonly used in literature (De Monte et al. 2006; Ishikawa and Komi 2004; Finni et al. 2001) to dissociate the behaviour of muscles fascicle and tendinous tissues, it has some limitations. The shortening velocity of knee extensor muscles was restricted to the shortening velocity of vastus lateralis muscle, considered as a surrogate of the knee extensor muscles. Although the muscular properties of vastus lateralis muscle are similar to vastus medialis and intermedius muscles, rectus femoris possesses specific properties (e.g., high proportion of fast-twitch fibres, muscular geometry). These characteristics indicate that rectus femoris muscle may play a substantial role in the achievement of high knee joint velocity. However, due to the complexity of the muscular geometry of rectus femoris, muscle fascicle tracking during dynamic contractions of this muscle represents a technical challenge (Blazevich et al. 2006; Massey et al. 2015). Regardless of this, we are confident that the vastus lateralis shortening velocity is the most representative of all knee extensors muscles behaviour. The estimation of the force produced by an individual muscle can be calculated in isometric condition using PCSA measurements and muscle moment arm (Baxter and Piazza 2014; Fukunaga et al. 1996). Thus, we considered in this work that the vastus lateralis was responsible for $\sim 34 \%$ of the total joint torque during knee extension (Akima et al. 1995). However, these approaches have necessarily some limitations for dynamic contractions. Indeed, individual muscle force-velocity relationship should fairly influence these contributions. Therefore, future studies should try to estimate the individual muscle force-velocity relationships to better understand the respective involvements of individual muscles during fast motions. Moreover, the estimation of the tendinous tissues changes in length which could be considered as an additional limitation of this model, as discussed in our previous studies (Hauraix et al. 2013, 2015).

In addition, the field of view of the probe used in this study (i.e., $55 \mathrm{~mm}$ ) was relatively small compared to the size of large muscles, such as the human vastus lateralis (Cronin and Lichtwark 2013). This required using an extrapolation of the visible part of the fascicle to estimate the whole length according to aponeuroses (Arampatzis et al. 2006; Blazevich et al. 2006). However, Finni et al. (2003) showed a relatively small error measurement, ranging between 2 and $7 \%$ of the vastus lateralis. Panoramic mode offered by ultrasound scanners allow to observe the entire muscle fascicle length (Noorkoiv et al. 2010). However, the use of this method is limited to the measurement performed at rest and during isometric contractions. An alternative method requires placing two ultrasound probes in series to observe the entire muscle fascicle 
length (Herbert et al. 2015). Further studies should appraise the error of measurement associated with muscle fascicle extrapolation using two probes placed in series to explore dynamic contractions.

\section{Conclusion}

This study contributes to extend our knowledge on muscle fascicles-tendon interactions during maximal concentric contractions. Muscle fascicles contribution to muscle-tendon unit shortening velocity of the vastus lateralis was constant up to the maximal knee extension velocity that subjects were able to achieve. Thus, the relation between muscle fascicle velocity and joint velocity was strongly fitted by a linear model. However, the contribution of muscle fascicles to the muscle-tendon shortening velocity was largely higher for the vastus lateralis (i.e., $83 \%$ on average) compared to the gastrocnemius medialis (Hauraix et al. 2015). The specific mechanical properties of vastus lateralis tendinous tissues could at least partly explain this greater participation of muscle fascicles. As a whole, our results demonstrated that the behaviour of muscle fascicle in vivo during maximal knee extension contractions strongly accorded with the classical Hill's modelling forcevelocity relationship and then allowed to confidently determine the maximal muscle shortening velocity. Based on the maximal shortening velocities reported in the present study, further studies should investigate the muscle fascicle behaviour during complex movements (e.g., jumping or running) to evaluate the link between velocity property of muscle fascicles and multi-joint performance.

Acknowledgements H Hauraix was supported by a scholarship funded by the French Ministry of Research. The authors thank all the participants for their involvement in the experiment and wish to extend their sincere appreciation to the French Institute of Sport (INSEP) for material assistance.

\section{Compliance with ethical standards}

Conflict of interest No conflicts of interest, financial or otherwise, are declared by the authors.

\section{References}

Akima H, Kuno S, Fukunaga T, Katsuta S (1995) Architectural properties and specific tension of human knee extensor and flexor muscles based on magnetic resonance imaging. Jpn J Phys Fit Sports Med 44:267-278. doi:10.7600/jspfsm1949.44.267

Arampatzis A, De Monte G, Karamanidis K, Morey-Klapsing G, Stafilidis S, Bruggemann GP (2006) Influence of the muscle-tendon unit's mechanical and morphological properties on running economy. J Exp Biol 209:3345-3357. doi:10.1242/jeb.02340
Astley HC, Roberts TJ (2012) Evidence for a vertebrate catapult: elastic energy storage in the plantaris tendon during frog jumping. Biol Lett 8:386-389. doi:10.1098/rsbl.2011.0982

Austin N, Nilwik R, Herzog W (2010) In vivo operational fascicle length of vastus lateralis during sub-maximal and maximal cycling. J Biomech 43:2394-2399. doi:10.1016/j. jbiomech.2010.04.016

Barany M (1967) ATPase activity of myosin correlated with speed of muscle shortening. J Gen Physiol 50(6):197-218

Baxter J, Piazza S (2014) Plantar flexor moment arm and muscle volume predict torque-generating capacity in young men. J Appl Physiol 116(5):538-544. doi:10.1152/japplphysiol.01140.2013

Benard MR, Becher JG, Harlaar J, Huijing PA, Jaspers RT (2009) Anatomical information is needed in ultrasound imaging of muscle to avoid potentially substantial errors in measurement of muscle geometry. Muscle Nerve 39:652-665. doi:10.1002/mus.21287

Biewener A, Baudinette R (1995) In vivo muscle force and elastic energy storage during steady-speed hopping of tammar wallabies (Macropus eugenii). J Exp Biol 198:1829-1841

Blazevich AJ, Gill ND, Zhou S (2006) Intra- and intermuscular variation in human quadriceps femoris architecture assessed in vivo. $\mathrm{J}$ Anat 209:289-310. doi:10.1111/j.1469-7580.2006.00619.x

Bobbert MF, Huijing PA, van Ingen Schenau GJ (1986) A model of the human triceps surae muscle-tendon complex applied to jumping. J Biomech 19:887-898

Chino K, Oda T, Kurihara T, Nagayoshi T, Yoshikawa K, Kanehisa H, Fukunaga T, Fukashiro T, Kawakami Y (2008) In vivo fascicle behavior of synergistic muscles in concentric and eccentric plantar flexions in humans. J Electromyogr Kinesiol 18:79-88. doi:10.1016/j.jelekin.2006.08.009

Cronin NJ, Lichtwark G (2013) The use of ultrasound to study muscle-tendon function in human posture and locomotion. Gait Posture 37:305-312. doi:10.1016/j.gaitpost.2012.07.024

Cronin NJ, Carty CP, Barrett RS, Lichtwark G (2011) Automatic tracking of medial gastrocnemius fascicle length during human locomotion. J Appl Physiol 111:1491-1496. doi:10.1152/ japplphysiol.00530.2011

Cronin NJ, Avela J, Finni T, Peltonen J (2013) Differences in contractile behaviour between the soleus and medial gastrocnemius muscles during human walking. J Exp Biol 216:909-914. doi:10.1242/jeb.078196

de Leva P (1996) Adjustments to Zatsiorsky-Seluyanov's segment inertia parameters. J Biomech 29:1223-1230

De Monte G, Arampatzis A, Stogiannari C, Karamanidis K (2006) In vivo motion transmission in the inactive gastrocnemius medialis muscle-tendon unit during ankle and knee joint rotation. J Electromyogr Kinesiol 16:413-422. doi:10.1016/j. jelekin.2005.10.001

Ettema GJ, van Soest AJ, Huijing PA (1990) The role of series elastic structures in prestretch-induced work enhancement during isotonic and isokinetic contractions. J Exp Biol 154:121-136

Farcy S, Nordez A, Dorel S, Hauraix H, Portero P, Rabita G (2014) Interaction between gastrocnemius medialis fascicle and Achilles tendon compliance: a new insight on the quickrelease method. J Appl Physiol 116(3):259-266. doi:10.1152/ japplphysiol.00309.2013

Finni T, Ikegawa S, Komi PV (2001) Concentric force enhancement during human movement. Acta Physiol Scand 173:369-377. doi:10.1046/j.1365-201X.2001.00915.x

Finni T, Ikegawa S, Lepola V, Komi PV (2003) Comparison of forcevelocity relationships of vastus lateralis muscle in isokinetic and in stretch-shortening cycle exercises. Acta Physiol Scand 177:483-491. doi:10.1046/j.1365-201X.2003.01069.x

Fontana Hde B, Roesler H, Herzog W (2014) In vivo vastus lateralis force-velocity relationship at the fascicle and muscle tendon 
unit level. J Electromyogr Kinesiol 24:934-940. doi:10.1016/j. jelekin.2014.06.010

Forrester SE, Yeadon MR, King MA, Pain MT (2011) Comparing different approaches for determining joint torque parameters from isovelocity dynamometer measurements. J Biomech 44:955961. doi:10.1016/j.jbiomech.2010.11.024

Fukunaga T, Roy RR, Shellock FG, Hodgson JA, Edgerton VR (1996) Specific tension of human plantar flexors and dorsiflexors. J Appl Physiol 80:158-165

Fukunaga T, Kubo K, Kawakami Y, Fukashiro S, Kanehisa H, Maganaris CN (2001) In vivo behaviour of human muscle tendon during walking. Proc Biol Sci R Soc 268:229-233. doi:10.1098/ rspb.2000.1361

Guilhem G, Doguet V, Hauraix H, Lacourpaille L, Jubeau M, Nordez A, Dorel S (2016) Muscle force loss and soreness subsequent to maximal eccentric contractions depend on the amount of fascicle strain in vivo. Acta Physiol 217:152-163. doi:10.1111/ apha. 12654

Hauraix H, Nordez A, Dorel S (2013) Shortening behavior of the different components of muscle-tendon unit during isokinetic plantar flexions. J Appl Physiol 115:1015-1024. doi:10.1152/ japplphysiol.00247.2013

Hauraix H, Nordez A, Guilhem G, Rabita G, Dorel S (2015) In vivo maximal fascicle-shortening velocity during plantar flexion in humans. J Appl Physiol 119:1262-1271. doi:10.1152/ japplphysiol.00542.2015

Herbert RD, Heroux ME, Diong J, Bilston LE, Gandevia SC, Lichtwark GA (2015) Changes in the length and three-dimensional orientation of muscle fascicles and aponeuroses with passive length changes in human gastrocnemius muscles. J Physiol 593:441-455. doi:10.1113/jphysiol.2014.279166

Hill AV (1938) The Heat of Shortening and the Dynamic Constants of Muscle. Proc R Soc London B Biol Sci 126:136-195

Ichinose Y, Kawakami Y, Ito M, Kanehisa H, Fukunaga T (2000) In vivo estimation of contraction velocity of human vastus lateralis muscle during "isokinetic" action. J Appl Physiol $88: 851-856$

Ishikawa M, Komi PV (2004) Effects of different dropping intensities on fascicle and tendinous tissue behavior during stretch-shortening cycle exercise. J Appl Physiol 96:848-852. doi:10.1152/ japplphysiol.00948.2003

Ishikawa M, Pakaslahti J, Komi PV (2007) Medial gastrocnemius muscle behavior during human running and walking. Gait Posture 25:380-384. doi:10.1016/j.gaitpost.2006.05.002

Johnson MA, Polgar J, Weightman D, Appleton D (1973) Data on the distribution of fibre types in thirty-six human muscles. An autopsy study. J Neurol Sci 18:111-129
Kawakami Y, Muraoka T, Ito S, Kanehisa H, Fukunaga T (2002) In vivo muscle fibre behaviour during counter-movement exercise in humans reveals a significant role for tendon elasticity. J Physiol 540:635-646

Konow N, Azizi E, Roberts TJ (2012) Muscle power attenuation by tendon during energy dissipation. Proc Biol Sci R Soc 279(1731):1108-1113. doi10.1098/rspb.2011.1435

Kubo K, Teshima T, Ikebukuro T, Hirose N, Tsunoda N (2014) Tendon properties and muscle architecture for knee extensors and plantar flexors in boys and men. Clin Biomech 29:506-511. doi:10.1016/j.clinbiomech.2014.04.001

Lichtwark GA, Barclay CJ (2010) The influence of tendon compliance on muscle power output and efficiency during cyclic contractions. J Exp Biol 213(5):707-714. doi:10.1242/jeb.038026

Lichtwark GA, Bougoulias K, Wilson AM (2007) Muscle fascicle and series elastic element length changes along the length of the human gastrocnemius during walking and running. J Biomech 40:157-164. doi:10.1016/j.jbiomech.2005.10.035

Massey G, Evangelidis P, Folland J (2015) Influence of contractile force on the architecture and morphology of the quadriceps femoris. Exp Physiol 100:1342-1351. doi:10.1113/ep085360

Noorkoiv M, Stavnsbo A, Aagaard P, Blazevich AJ (2010) In vivo assessment of muscle fascicle length by extended field-of-view ultrasonography. J Appl Physiol 109:1974-1979. doi:10.1152/ japplphysiol.00657.2010

Osternig LR (1986) Isokinetic dynamometry: implications for muscle testing and rehabilitation. Exerc Sport Sci Rev 14:45-80. doi:10.1249/00003677-198600140-00005

Randhawa A, Jackman ME, Wakeling JM (2013) Muscle gearing during isotonic and isokinetic movements in the ankle plantarflexors. Eur J Appl Physiol 113:437-447. doi:10.1007/ s00421-012-2448-z

Thorstensson A, Larsson L, Tesch P, Karlsson J (1977) Muscle strength and fiber composition in athletes and sedentary men. Med Sci Sports 9:26-30

Tihanyi J, Apor P, Fekete G (1982) Force-velocity-power characteristics and fiber composition in human knee extensor muscles. Eur J Appl Physiol 48:331-343

Visser JJ, Hoogkamer JE, Bobbert MF, Huijing PA (1990) Length and moment arm of human leg muscles as a function of knee and hip-joint angles. Eur J Appl Physiol 61:453-460

Wickiewicz TL, Roy RR, Powell PL, Perrine JJ, Edgerton VR (1984) Muscle architecture and force-velocity relationships in humans. J App Physiol 57(2):435-443

Wilson AM, Watson JC, Lichtwark GA (2003) Biomechanics: a catapult action for rapid limb protraction. Nature 421:35-36. doi: $10.1038 / 421035 \mathrm{a}$ 